\title{
Reglas sociales y comportamiento prudencial en la teoría laboral del valor
}

\author{
Social rules and prudential behaviour \\ in labour theory of value
}

\author{
César Rendueles \\ Universidad Complutense de Madrid
}

Recibido: 02/09/2010

Aceptado: 25/01/2011

\section{Resumen}

En la teoría económica marxiana el valor es una magnitud subyacente. Por eso los críticos de Marx han denunciado sistemáticamente que se trata de una hipótesis metafísica sin significado empírico. Este texto propone una doble estrategia de justificación. En primer lugar, replantea el concepto el valor en términos intensionales, como un agregado social relacionado con una norma colectiva. En segundo lugar, analiza el problema de la transformación como un teorema de imposibilidad.

Palabras clave: teoría laboral del valor, reglas sociales, dilema del prisionero, intensionalidad.

\begin{abstract}
In marxian economic theory value is an underlying magnitude. Hence Marx's critics have denounced it systematically as a metaphysical hypothesis. This essay suggests a twofold justification strategy. First, puts forward value in intensional terms, as a social collection related to a social rule. Second, analyzes transformation problem as an impossibility theorem.
\end{abstract}


Keywords: labour theory of value, social rules, prisoner's dilemma, intentionality.

Cualquiera que haya dedicado algún tiempo al estudio de la disputa secular sobre la teoría laboral del valor de Marx no puede sino recibir con desconfianza una nueva intervención en un terreno roturado hasta el agostamiento y que ha alcanzado espeluznantes cotas de sofisticación formal. Quienes tengan la fortuna de no haber padecido tal experiencia tardoescolástica recibirán la propuesta con el gesto de ironía que se reservaría para alguien que siguiera discutiendo la teoría del flogisto. Lo que pretendo mostrar a continuación no es tanto la posibilidad, en la que no creo, de mediar filosóficamente sobre una discusión científica que, además, considero infructuosa, cuanto el modo en que el análisis de la esterilidad de ese debate en particular tiene consecuencias interesantes para la filosofía de las ciencias sociales en general.

\section{Reglas y conducta prudencial}

Una de las claves hermenéuticas de la obra de Marx es la idea de que la realidad social no es comprensible en su manifestación más inmediata, sino que existe alguna suerte de núcleo estable de inteligibilidad -al que Marx se refiere a menudo sin ambages como "esencia"- que explica el devenir de las comunidades humanas. Una forma de expresar esta tesis de un modo menos esotérico y, sobre todo, menos comprometido gnoseológicamente pasa por su reducción a relaciones entre conjuntos de reglas sociales.

Uno de estos agregados de reglas demarca un espacio regido por el juego de la oferta y la demanda característico de las relaciones mercantiles. Estas reglas son muy idiosincrásicas porque, en principio, apenas agregan nada a la estructura misma de la conducta prudencial individual. Un ser racional que careciera por completo de vida social - un programa de ordenador, por ejemplo- podría ceñirse a este tipo de comportamiento. En cambio, las reglas sociales típicas se caracterizan por su densidad intersubjetiva, hasta el punto de que se suelen considerar irreductibles a conducta prudencial 1 . Así que, en realidad, es motivo de controversia si cabe

\footnotetext{
1 Jon Elster explica la contraposición entre conducta prudencia y reglas sociales con una paradoja. Supongamos que Juan está dispuesto a pagar un máximo de diez euros a un chico para que le limpie el coche. Si el limpiador le exigiera once euros preferiría dedicar media hora a limpiar su coche él mismo. Supongamos que un vecino bromista le ofrece a Juan veinte euros a cambio de que limpie su coche. Juan se niega indignado. Al no querer pagar los once euros que le pide el chico, el hombre manifiesta implícitamente que media hora de su tiempo vale a lo sumo once euros. Al rechazar la pro-
} 
hablar de los comportamientos mercantiles en términos de reglas -en la medida en que su generalización podría ser una transformación histórica reciente- $\mathrm{o}$, más bien, forman parte del horizonte de comprensión de cualquier conducta racional. Una tercera opción, muy difundida, propone la existencia de dos regímenes pragmáticos que mantienen una convivencia tumultuosa: de un lado el ámbito de la racionalidad instrumental individual, de otro, el de las reglas sociales autónomas.

Marx postula la existencia de una relación explicativa entre el contexto pragmático mercantil -cuyas reglas son transparentes y generalmente se pueden reducir a expresiones imperativas que cualquier agente racional está en condiciones de entender ("compre barato y venda caro" y cosas por el estilo)-y otro tipo de reglas sociales relacionadas con la producción que a) tienen contenidos intencionales muy opa$\cos , \mathrm{b})$ son más informativas que las acciones instrumentales, ya sea por su mayor estabilidad o por cualquier otra razón y c) no son inmediatamente coherentes con la conducta mercantil. Una de estas reglas de segundo tipo sería la "ley del valor", esto es, la idea de que el criterio que regula el intercambio y, por consiguiente, la supervivencia material de la sociedad moderna es cierta cantidad mínima de trabajo que se necesita para producir cada una de las mercancías.

La teoría del valor no describe la intención consciente de quienes acuden al mercado, que sólo se rigen por la regla del intercambio de equivalentes y son ciegos a cualquier otro principio inmanente. El valor no es una norma de conducta sino una regla más profunda que se manifestaría a través de la institución mercantil. Un buen y un mal símil son las reglas lingüísticas que subyacen a las locuciones pragmáticas cotidianas en cualquier idioma. Es una buena comparación porque la sintaxis sólo se realiza en las expresiones de hablantes que generalmente desconocen esa estructura. Es mala porque el problema no es sólo que quienes acuden al mercado desconozcan la ley del valor, cuanto que, como queda de manifiesto en el problema de la transformación, ésta entra en tensión con sus intenciones manifiestas. Un poco como si mi propósito fuera que el sujeto concuerde con el objeto directo pero una fuerza misteriosa me obligara sistemáticamente a que concordara con el verbo.

El punto de partida de la teoría laboral del valor marxiana es elegante. La práctica cotidiana de igualar dos valores de uso diferentes a través del intercambio implicaría que ambos tienen algo en común. Si se acepta que el único atributo cuantificable que comparten todas las mercancías es que son productos del trabajo humano, cabe plantear la existencia de una magnitud absoluta subyacente al valor de cambio. Dicha magnitud subyacente estaría determinada por el tiempo de trabajo "socialmente necesario" que se requiere para producir la mercancía en cuestión,

posición del otro vecino que le ofrece veinte euros, manifiesta implícitamente que esa media hora vale por lo menos veinte euros, pero no puede valer menos de once euros y más de veinte euros. Lo que hace que en un caso Juan otorgue tanta importancia a un euro y en otra tan poca a veinte es que en un caso se ciñe a una conducta prudencial y en otro sigue una regla social. 
esto es, no por el tiempo de trabajo materializado en ella sin más, sino por alguna clase de magnitud ideal coherente con el nivel de desarrollo de las fuerzas productivas y la competencia mercantil. El problema es, por supuesto, que los consumidores y vendedores únicamente están preocupados por maximizar sus ganancias y minimizar sus pérdidas y, de este modo, son perfectamente ajenos a esa supuesta ley. Dado que el valor no juega ningún papel pragmático en la conducta individual, ¿qué nos permite afirmar que no es sencillamente una cifra? Evidentemente podemos establecer una media de cualquier conjunto de números pero eso no significa que exprese una magnitud absoluta con algún significado preciso. El problema, en resumidas cuentas, puede enunciarse así: ¿cómo ocurre materialmente que los individuos que acuden al mercado respetan una regla, el valor, que no sólo no está presente en su vida intencional, sino que parece entrar en conflicto con ella? En los últimos tiempos la respuesta más popular a esta pregunta ha sido que simplemente no ocurre: no existe ninguna ley del valor. Otro conjunto de respuestas han intentado esquivar el problema encontrando un procedimiento matemático o filosófico que explique la relevancia (matemática o filosófica) de la norma esencial y su congruencia (matemática o filosófica) con la conducta individual.

A continuación propondré una doble vía de intervención. De un lado, trataré de contribuir al esclarecimiento de la forma general de la justificación que cabría dar de la existencia del valor como algo más que una cifra o un análisis filosófico. En segundo lugar, intentaré mostrar por qué cierto tipo de fracaso inevitable de la ley del valor puede ser filosóficamente fructífero.

\section{Una interpretación no extensional de la ley del valor}

El problema al que, en el fondo, remiten todos los críticos más agudos de la teoría del valor es lo insatisfactorio que resulta un concepto, como el valor, que opera como un todo a espaldas de la conducta individual, según una estrategia típicamente funcionalista. La tesis de Marx sería análoga a la de aquellos sociólogos que consideran que se puede explicar la existencia de, por ejemplo, una religión apelando a sus efectos estabilizadores sobre una comunidad sin dar cuenta de cómo intervienen en ese proceso los individuos que la componen. El dilema que plantean los críticos es que o bien el fenómeno agregado es sencillamente el resultado de la interacción de sus elementos, de modo que su redescripción funcionalista sería redundante, o bien surge al margen de ellos, en cuyo caso se trata de una tesis metafísica. G. A. Cohen depuró al máximo esta argumentación detectando una incoherencia en la versión estricta de la teoría laboral del valor marxiana:

Supóngase que una mercancía tiene cierto valor en un momento t. Entonces ese valor, dice la teoría laboral, está determinado por el tiempo de trabajo socialmente necesario 
requerido para producir una mercancía de ese tipo. Preguntémonos ahora: ¿requerido para producirlo cuándo? La respuesta es: en t, en el momento en el que tiene el valor que hay que explicar. La cantidad de valor requerido para producirlo en el pasado y, $a$ fortioti, la cantidad de tiempo realmente gastado al producirlo son magnitudes estrictamente irrelevantes para su valor, si la teoría laboral del valor es verdadera [...]. La teoría supone que el trabajo pasado es irrelevante para establecer cuánto valor tiene ahora una mercancía. Pero el trabajo pasado no sería irrelevante si creara el valor de la mercancía. En consecuencia, si la teoría del valor trabajo es verdadera, entonces el trabajo no crea valor ${ }^{2}$.

Una forma razonable de responder a la crítica de Cohen pasa por una defensa de las características no extensionales del valor en la formulación de Marx. Así se disolvería el dilema planteado más arriba demostrando que, en realidad, su segundo término es una opción ontológicamente aceptable y no necesariamente metafísica. En este sentido, cabría entender las mercancías, esto es, las entidades que tienen "valor", como "agregados sociales", como una forma de entidad social. Entre las más rigurosas sistematizaciones de las condiciones de posibilidad de las entidades sociales se cuenta el colectivismo de Ruben, quien define los agregados sociales como una colección unida por alguna propiedad común de índole social (en este caso el "valor") 3 . Los grupos existen cuando los individuos con ciertas propiedades en común son considerados o se consideran a sí mismos de ciertos modos socialmente significativos. Así que, en última instancia, el único criterio de identidad de un agregado social sería la existencia de una práctica general, una regla en virtud de la cual ciertos individuos son identificados de una manera socialmente significativa por esas propiedades que comparten. Por supuesto, la debilidad principal de esta hipótesis colectivista es que se puede mantener con coherencia que una norma no es más que una creencia o una actitud compartida por individuos. Precisamente, la teoría del valor de Marx propone una regla práctica general que, sin embargo, no puede reducirse a una creencia compartida.

La argumentación de Cohen demuestra que esta regla está indeterminada extensionalmente. Desde el punto de vista de los partidarios de una ontología social colectivista, se trata de una aporía menor, ya que en general la identidad de los grupos sociales que postulan no se puede reducir a los elementos que los componen. En efecto, se suele hablar de los grupos sociales como entidades no extensionales porque, a diferencia de lo que ocurre con los conjuntos matemáticos, dos grupos no idénticos pueden tener exactamente los mismos miembros y porque la identidad de un grupo social se preserva aunque se produzcan cambios en los miembros. Por ejemplo, la entidad Francia no puede ser identificada con sus habitantes ni con su

2 Cohen, G. A.: "The Labour Theory of Value and the Concept of Exploitation" en I. Steedman (ed.), The Value Controversy, Londres, Verso, 1981, p. 209.

3 Ruben, D.-H.: The Metaphysics of the Social World, Londres, Routledge \& Kegan Paul, 1985, p. 21. 
ubicación geográfica. Para muchos filósofos, la no extensionalidad de estos grupos demuestra su inexistencia o, al menos, su futilidad científica. Sin embargo, es indudable que esta clase de fenómenos se pueden verificar en nuestras prácticas lingüísticas y gnoseológicas cotidianas. Es posible que estas prácticas sean meramente ideológicas y que los conocimientos que extraemos de ellas sean espurios pero ese es un asunto distinto: en el peor de los casos las teorías que utilicen esta clase de entidades serán sociología, economía o psicología popular cuya inutilidad general dependerá de que exista una alternativa científica más respetable.

En suma, tal vez se podría concebir el valor de una mercancía como un agregado social (de horas de trabajo) cuya identidad viene dada por una regla pública. De este modo, se salvaría la incongruencia que señala Cohen. La variación en la composición temporal de una mercancía no afectaría necesariamente a su identidad ni entrañaría de suyo una contradicción. Una objeción fácil a este argumento es que precisamente el problema es que no existe una regla pública que permita la identificación de las mercancías a partir de la propiedad "ser el producto de cierta cantidad de horas de trabajo socialmente necesarias para su producción". En el mejor de los casos se trataría de una regla meramente posible que los individuos desconocen. La única respuesta posible a esta objeción, y posiblemente la más cercana a los planteamientos de Marx, pasa por sostener que esa regla es una inferencia plausible que los agentes sociales implicados en estas prácticas podrían realizar.

Y lo cierto es que en algunas ocasiones significativas, esa regla implícita y susceptible de descubrimiento inferencial se vuelve explícita a través de una especie de proceso gnoseológico colectivo. De manera recurrente, en los momentos en que tienen lugar los procesos institucionales que regularizan las prácticas mercantiles -típicamente en momentos fundacionales o en las situaciones de crisis- salen a la luz reglas pragmáticas explícitas relativamente próximas a la teoría del valor. Por ejemplo, las polémicas históricas, muy activas en la actualidad, acerca de la consideración jurídica de la propiedad intelectual, al igual que las críticas a la especulación financiera e inmobiliaria, muestran que existen procesos inferenciales relativamente cercanos a la teoría laboral del valor con algún arraigo en la cultura contemporánea.

En términos generales, y dicho de un modo muy impresionista, las legislaciones modernas de la propiedad intelectual evolucionaron desde sistemas de base puramente mercantil a una estructura jurídica que buscaba un equilibrio entre la protección de los intereses de los autores, los productores y el público. Las transformaciones tecnológicas de las últimas décadas han cuestionado este sistema. En aquellos medios de comunicación de masas en los que el coste marginal de cada nuevo uso tiende a cero, los productores cobran por productos virtualmente gratuitos. Desde la perspectiva de muchos grupos de usuarios, estos cambios han acercado la industria cultural al capital ficticio y han quebrantado el espíritu de la ley, que 
vincula la explotación económica de la propiedad intelectual a una función social que, ahora, puede ser alcanzada sin grandes inversiones. En última instancia, los argumentos de quienes defienden esta última posición se basan en la idea de que las fuentes de beneficio meramente especulativas son parasitarias de las productivas y cuestionan la legitimad de cobrar por una mercancía cuya producción no requiere tiempo de trabajo humano. Se trata de un razonamiento que no se limita a la industria cultural, sino que tiene importantes simetrías en el caso de las patentes biológicas, que a menudo se basan en una mera labor administrativa.

Parece, así, que sería al menos teóricamente posible encontrar una regla social que cuestione la legitimidad de aquellos beneficios que se apartan significativamente de cierto "centro de gravedad", por emplear la expresión de Marx. Y eso con independencia de que los conflictos relacionados con la propiedad intelectual resulten convincentes en este sentido. La comprensión de esa regla depende de un proceso inferencial conflictivo -en tensión con otro conjunto de normas mercantiles-, por eso es razonable centrar la atención en momentos críticos en los que los límites de la reproducción material de la economía capitalista resultan más visibles. Evidentemente, nada de esto nos indica que ese núcleo de gravedad esté determinado por la ley del valor (ni siquiera está muy claro cómo se podría evaluar empíricamente una tesis tal), pero al menos sugiere la existencia de una regla social compatible con ella que, así, permite cuestionar las críticas basadas en argumentos extensionalistas radicales. Por eso, se trata de un resultado mucho más interesante desde el punto de vista de la filosofía de las ciencias sociales que de la economía, pues parece mostrar que la estrategia colectivista no es meramente redundante. Si la argumentación precedente fuera válida, el colectivismo no describiría de un modo innecesariamente prolijo fenómenos agregados que se pueden reducir a creencias y deseos individuales, sino que sacaría a la luz realidades sociales -como la ley del valor- que sólo son visibles en un contexto holista. Claro que también podría tratarse de un círculo vicioso, una relación de complicidad nefasta entre el limbo colectivista y el infierno de la teoría del valor.

\section{Los precios de producción}

Todo lo anterior es sólo la mitad del problema de la teoría del valor, y de hecho, la más fácil de sobrellevar. Su auténtica debilidad no es tanto que la teoría del valor sea gnoseológicamente invisible cuanto que el proceso inferencial que entraña es pragmáticamente ineficaz por lo que toca al comportamiento mercantil individual, que es justamente lo que debería explicar. Aunque los individuos arrojados al juego de la oferta y la demanda sean conscientes de los procesos de valorización que supuestamente regulan su actividad, ese conocimiento en nada afectaría a su com- 
portamiento en el mercado. Es decir, aunque aceptásemos la congruencia de la ley del valor y su validez empírica, aún quedaría por explicar en qué sentido es explicativa, es decir, su relación material con las conductas prudenciales. Por supuesto, el lugar clásico donde se ha planteado este problema es en el análisis de la transformación de los valores en precios de producción, que ha dado lugar a un largo debate que se remonta a los principios del siglo XX.

Según Marx, si las mercancías se vendieran a su valor, el plusvalor generado en una industria redundaría directamente en beneficio de los capitalistas de esa industria. De modo que las industrias que emplean poca maquinaria y mucha mano de obra obtendrían beneficios mayores que las que emplean menos mano de obra. Obviamente no ocurre así. La tasa de ganancia es más o menos igual en todas las industrias o, al menos, es independiente de la composición de sus capitales. La respuesta de Marx a este problema consiste en afirmar que el plusvalor total de una sociedad se reparte equitativamente entre todas las ramas de la industria. Pero para que sea posible esta redistribución de la ganancia, los precios de producción tienen que diferir de los valores. Los precios de producción se calculan añadiendo a los costes totales la parte proporcional del plusvalor total. En aquellas industrias donde se emplee poca mano de obra los precios de producción estarán por encima de los valores. Marx ejemplifica esto con una famosa tabla de cinco industrias con diferentes composiciones orgánicas de capital que se reparten el plusvalor total a través de una ganancia media que añaden a sus precios de coste para, así, determinar el precio de producción. Según Marx: "Puesto que el valor global de las mercancías regula el plusvalor global, mientras que éste, en cambio, regula el nivel de la ganancia media y por lo tanto de la tasa general de ganancia -en cuanto ley general o como ley que rige las oscilaciones-, entonces la ley del valor regula los precios de producción" 4 . Lo que intenta subrayar Marx es que son las condiciones de producción las que establecen las condiciones generales de la ganancia, con independencia de cuál sea el método por el cuál se distribuya entre los capitalistas.

Como es sabido, el problema del enfoque de Marx es que en la tabla de industrias los precios de coste a los que se añade la ganancia media se calculan en términos de valor. Sin embargo, parece evidente que muchos de los elementos utilizados en la producción se adquieren en el mercado y por lo tanto, deberían expresarse ya en precios de producción. Según Meek, "mientras los valores de las mercancías que forman el output del sistema se transforman debidamente en precios de producción como resultado de la redistribución de la suma dada de plusvalores, los valores de las mercancías que forman el input del sistema se dejan sin transformar. Este método sólo tendría sentido si todos los inputs cuyos valores constituyen el precio de costo fueran producidos por capitales de composición orgánica media, o si fueran

${ }^{4}$ Marx, K.: El capital. Crítica de la economía política, Libro III, vol. 6, Madrid, Siglo XXI, 1979, p. 228. 
producidos fuera del sistema y, por una u otra razón, adquiridos a su valor"5. Este problema se ha considerado la clave de la refutación de la teoría económica de Marx. Por eso sus defensores han propuesto numerosos procedimientos formales que tratan de establecer la interdependencia de inputs y outputs. Pero el problema no es matemático. No se trata de transformar unos números en otros, sino de mostrar cómo una regla social sin contenido intencional -el valor- se relaciona con lo que el propio Marx considera los engranajes conductuales reales y observables en el mercado6.

\section{Las "soluciones" al dilema del prisionero}

Con amplias dosis de generosidad hermenéutica, el problema de la transformación de los valores en precios de producción puede ser entendido metafóricamente como una especie de inversión del dilema del prisionero. El dilema del prisionero muestra los límites de la racionalidad prudencial en un contexto social. En cambio, el problema de la transformación muestra los límites del colectivismo y la sociabilidad en el capitalismo.

Las interacciones estratégicas, como la que caracteriza la situación que describe el problema de la transformación, se pueden considerar desde dos puntos de vista distintos. Desde el punto de vista de la utilidad que reporta, hablamos de la optimidad de una estrategia: el resultado de una interacción es un óptimo de Pareto para un individuo cuando no hay ningún otro resultado que le reporte mayor utilidad sin que disminuya la utilidad de otro individuo. Desde el punto de vista de la coordinación de estrategias, decimos que dos estrategias están en equilibrio si ninguno de los jugadores puede mejorar su situación mediante una modificación unilateral de su posición. La optimidad y el equilibrio no siempre son compatibles. Precisamente el dilema del prisionero describe un juego cooperativo con un único punto de equilibrio que es subóptimo. En el dilema del prisionero, según la descripción de Robert Axelrod, "hay dos jugadores. Cada uno de ellos puede optar entre dos alternativas, cooperar o no hacerlo. Cada uno de ellos tiene forzosamente que elegir sin saber lo que va a hacer el otro. Independientemente de lo que el otro pueda hacer, la defección proporciona siempre un resultado mejor que la cooperación. El problema es que si ninguno coopera ambos saldrán peor parados que si hubieran cooperado"7.

\footnotetext{
5 Meek, R. L.: Smith, Marx y después, Madrid, Siglo XXI, 1980, p. 131.

6 En palabras de Marx: "El capitalista individual (o la totalidad de los capitalistas de cada esfera particular de la producción), de mirada corta, tiene razón al creer que su beneficio no procede exclusivamente del trabajo empleado por él o por su rama. Eso es perfectamente verdad por lo que hace a su beneficio medio" (El capital, Libro III, cap. 9).

7 Axelrod, R.: La evolución de la cooperación, Madrid, Alianza, 1996, p. 19.
} 
Al igual que en el problema marxiano de la transformación, tampoco el dilema del prisionero es un problema gnoseológico. No sirve de nada saber cuál es la solución óptima: dada la estructura del juego, no se puede atribuir a los individuos estrategias intencionales que les lleven a optar por ese resultado.

La importancia del dilema del prisionero es que saca a la luz una limitación importante de cierto tipo de concepción de la naturaleza humana y de las ciencias sociales extremadamente influyente. Plantea un teorema de imposibilidad frente a la pretensión de reconstrucción atomística de la realidad social a partir de la interacción individual y recoge la incapacidad de la teoría de la decisión racional para explicar una infinidad de situaciones reales cooperativas. De alguna manera, señala qué es lo que hay que añadir a la conducta individual para que exista sociabilidad.

Hay dos formas muy conocidas de disolver, antes que resolver, el dilema del prisionero. La primera consiste simplemente en recordar que todo individuo piensa a menudo en términos de "nosotros". No es necesario justificar la sociabilidad. Los seres humanos somos animales gregarios y ese hecho precede a cualquier análisis de la interacción entre individuos. En segundo lugar, los intentos de solución más famosos estudian dilemas del prisionero iterativos. Muchas veces tomamos decisiones sabiendo que nos vamos a volver a encontrar con las personas con las que interactuamos, elegimos tomando en consideración su actuación pasada y los resultados futuros. De este modo, según la importancia que otorguemos a las próximas interacciones, podríamos optar por una estrategia cooperativa poco inteligente a corto plazo pero óptima a largo plazo. Esta dinámica dota a los jugadores de razones prudenciales a largo plazo para cooperar a corto plazo. En una archicitada investigación, Axelrod demostró que la estrategia más eficaz para fomentar la cooperación es extremadamente sencilla. Se conoce como "toma y daca" y consiste en comenzar cooperando, responder a la falta de cooperación con falta de cooperación, pero volver a cooperar inmediatamente sin mostrarse "rencoroso"".

En rigor, las estrategias iterativas no solucionan el dilema del prisionero. El jugador que adopta "toma y daca" para fomentar la cooperación tiene que tener una razón inicial para ser el primero en cooperar que no estaba dada en la estructura del juego. La virtud de la estrategia "toma y daca" es que permite que el otro entienda con facilidad que el primero tiene está disposición a cooperar. Por eso, la solución iterativa al dilema del prisionero puede interpretarse como el surgimiento de una forma de comunicación primitiva cuyo éxito depende precisamente de su claridad. El tipo de comunicación sin contenido intencional que se establece con las estrategias iterativas recuerda a esa clase de compromiso al que generalmente nos referimos como sociabilidad y que consiste en la capacidad para ver las intersecciones de preferencias individuales como algo más que la suma de sus partes. Lo que hace la

8 Axelrod, R.: La evolución de la cooperación, Madrid, Alianza, 1996, p. 60. 
estrategia iterativa es reducir al mínimo los requisitos de sociabilidad necesarios para disolver el dilema, localiza un grado cero de la sociabilidad - una comunicación mínima, con un contenido intencional infinitesimal- pero no puede renunciar a ella.

\section{Las "soluciones" al problema de la transformación}

Al margen de las soluciones puramente aritméticas, existen dos soluciones al problema de la transformación que tienen cierto aire de familia con los dos conjuntos principales de soluciones al dilema del prisionero. En primer lugar, se ha mantenido que no hay necesidad de realizar ninguna transformación puesto que "la transición del Libro I al Libro III [de El capital] no es una transición de valores-trabajo a precios, es una transición de precios agregados a precios individuales; así que Marx no se olvidó de transformar los inputs" 9 . Desde esta perspectiva, para Marx los valores serían metodológicamente anteriores a los precios, en la medida en que expresan la dimensión social de la producción, el intercambio y la desigualdad y su relación con la subsistencia material de una comunidad.

En segundo lugar, existe una solución iterativa al problema de la transformación desarrollada por Anwar Shaikh y que consiste en repetir el procedimiento de Marx corrigiendo en cada ocasión los inputs de cada elemento productivo según el multiplicador que se ha hallado en la anterior jugada. Si el resultado de dividir el output (expresado en precios) en el sector que produce los medios de producción entre el input global (expresado en valores) de capital constante da una desviación de, por ejemplo, 1'089, en la siguiente "jugada" habrá que multiplicar por esa cifra los distintos inputs de capital constante y así sucesivamente hasta que el multiplicador sea igual a 1 en todas las ramas industriales 10 .

La tabla de precios inicial presentada por Marx expresa el paso de una red de comunicación sui generis - un sistema estructurado de reglas sociales- a una situación de incomunicación competitiva. Es, en ese sentido, un dilema. Shaikh demuestra que ambas situaciones son pragmáticamente (y no sólo numéricamente) congruentes si se entienden como los extremos de una sucesión de operaciones repetidas. Es decir, dadas ciertas premisas se puede explicar el paso de un contexto normativo a otro en la que la información acerca de los valores carece de eficacia conductual. Es precisamente la situación inversa a la de un dilema del prisionero iterativo. De nuevo, lo crucial aquí no es el contenido intencional de los participantes en

\footnotetext{
9 Moseley, F.: Marx's Method in Capital. A Reexamination, New Jersey, Humanities Press, 1993, p. 176.

10 Cf. Shaikh, A.: "Marx's Theory of Value and the Transformation Problem" en J. Schwartz (ed.), The Subtle Anatomy of Capitalism, Santa Monica, Goodyear, 1977.
} 
el proceso, es decir, lo que conocen o dejan de conocer, sino qué decisiones prudenciales están en condiciones de tomar.

En rigor, la propuesta de Shaikh no resuelve el problema de la transformación, pues no aclara en qué sentido son entonces explicativos los valores sobre los precios de producción. Más bien parece poner de manifiesto el modo en que los procesos de mercantilización implican una destrucción de las reglas sociales características de los contextos productivos tradicionales, de la economía como proceso institucional, por emplear la expresión de Polanyi. Esto hace justicia a la grandeza de la teoría del valor y a su fuente principal de aporías. Marx se planteó una tarea imposible: trató de simultanear el análisis de reglas sociales antropológicamente densas, útiles para comprender los procesos de explotación en las sociedades capitalistas, con el estudio del comportamiento atomístico característico de los mercados. De algún modo, identificó una cierta continuidad entre el capitalismo y el resto de sistemas sociales históricos. A pesar de todo, parece decir Marx a menudo, incluso el capitalismo es una sociedad con alguna clase de estructura normativa. Una sociedad, sin embargo, que se concibe a sí misma como un agregado de individuos e insiste en ignorar las realidades antropológicas duraderas. El punto crucial es que Marx trató de interpretar esa sociedad en su propios términos, no renunció a la perspectiva emic y se vio abocado a una tensión permanente entre la búsqueda de la estructura de reglas sociales estables propia del capitalismo y el nivel fenoménico de autocomprensión de los agentes económicos, que desafía permanentemente la primera perspectiva. De ahí tanto esa irrepetible mezcla de géneros y estrategias característica de El capital como algunas de sus aporías más desafiantes.

César Rendueles

Departamento de Sociología V (Teoría Sociológica)

Facultad de Ciencias Políticas y Sociología

Universidad Complutense de Madrid crendueles@gmail.com 